\title{
Edge detection algorithm for omnidirectional images, based on superposition laws on Blach's sphere and quantum entropy
}

\author{
Ayoub Ezzaki ${ }^{1}$, Dirar Benkhedra ${ }^{2}$, Mohamed El Ansari $^{3}$, Lhoussaine Masmoudi ${ }^{1}$ \\ ${ }^{1}$ LCS laboratory, Physics Dept. Faculty of science, Mohammed V University in Rabat, Morocco. \\ ${ }^{2}$ CeReMAR Center, Mathematic Dept. Faculty of science, Mohammed V University in Rabat, Morocco \\ ${ }^{3}$ Informatics and Applications Laboratory, Computer Science Dept., Faculty of Science, My Ismail University in \\ Meknes, Morocco
}

Received $14^{\text {th }}$ of October 2020; accepted $21^{\text {st }}$ of February 2021

\begin{abstract}
This paper presents an edge detection algorithm for omnidirectional images based on superposition law on Bloch's sphere and quantum local entropy. Omnidirectional vision system has become an essential tool in computer vision, due to its large field of view. However, classical image processing algorithms are not suitable to be applied directly on this type of images without taking into account the spatial information around each pixel. To show the performance of the proposed method, a set of experimentation was done on synthetic and real images devoted to agriculture applications. Later, Fram \& Deutsh criterion has been adopted to evaluate its performance against three algorithms proposed in the literature and developed for omnidirectional images. The results show a better performance in term of edge quality, edge community and sensibility to noise.
\end{abstract}

Key Words: Edge detection, Omnidirectional images, Quantum image processing, Quantum entropy

\section{Introduction}

Omnidirectional images represent the type of images that covers almost the entire sphere or at least a full horizontal plane, therefore containing much more information than the conventional one. Duo to its various advantages, omnidirectional images has been widely used in several research fields such as: Person detection and tracking [1], robot navigation [2], 3D scene reconstruction [3], line image extraction [4] etc. essentially for the $360^{\circ}$ field of view provided by a such image. This large field of view can be reached by numerous technics notably: A rotating camera [5], a fish eye camera [6] and also with a combination between mirror and perspective camera: catadioptric camera [7]-[9].

Even with all the positive points, the major weakness with this type of images is the difficulty of using classical image processing algorithms directly on it, due to several reasons such as: the radial distortions provided by the geometry of omnidirectional image, the non-uniformity of the image resolution, etc. For that, a significant number of research interested on solving this issue [10]-[13], by proposing new algorithms which take into account the spatial information of the image. One of the most affected type of image processing algorithms is edge detection, duo to the use of kernels and convolution in the majority of the known methods.

Edge detection can be defined as the detection of significant and abrupt changes in grey level image intensity, the major aim is to detect important objects/events in the processed image, and its used in several

Correspondence to: ayoub_ezzaki@um5.ac.ma

Recommended for acceptance by Angel D. Sappa

https://doi.org/10.5565/rev/elcvia.1338

ELCVIA ISSN: 1577-5097

Published by Computer Vision Center / Universitat Autonoma de Barcelona, Barcelona, Spain 
applications linked to omnidirectional images: 3D human tracking [14], robot navigation monitoring system [15], Global Localization for Aerial Robots [16] etc. Duo to its importance, many researches has been carried out to propose new edge detection algorithms for omnidirectional images. In [17] the authors proposed a spherical gradient operator that computes the gradient from a discrete spherical image with irregular pixels. The authors in [18] proposed to define catadioptric image processing from geodesic metric in the unitary sphere, in order to simply adapt classical image processing methods to omnidirectional images. Domonceaux $\&$ al proposed in [19] to define a spherical neighborhood for each pixel in order to adapt Markovian methods to omnidirectional images. Likewise, authors in [20] used spherical harmonics to define convolution products in spherical images. Another work proposed by Hara \& al in 2015 purpose an extension of the Laplacien operator that can handle spatial inhomogeneity in omnidirectional images, by using a specially varying filter matrix [21]. Whereas the majority of edge detection algorithms for omnidirectional images presented in the literature are not designed directly on the spherical space which is more suitable for this image types.

In the last decades, the quantum image processing (QIP) theory was developed, based on quantum information theory, to overcome classical image processing algorithms' limits. As we will see later, it's come down to present the image information with a combination of all its states by the so-called quantum superposition law [22].

In this paper, we propose an algorithm for edge detection in omnidirectional images based on quantum entropy and superposition laws by spherical neighborhood definition on Bloch's sphere. The proposed algorithm avoids the binarisation step which is a cost consuming step in the majority of edge detection algorithms while taking into account the spatial information of the omnidirectional image in order to avoid the weakness of the classical methods. The proposed algorithm is composed of 3 major steps: i) Spherical neighborhood definition on Bloch's sphere. ii) Edge enhancement by quantum superposition iii) Edge localization using local quantum entropy.

In order to test our proposal regarding other algorithms presented in the literature, a set of experiments are carried out using synthetic and real images devoted to agriculture applications, after that we used Fram and Deutsh metrics presented in [23], to evaluates its performance in term of edge continuity and sensibility to noise.

The paper is organized as follow: section 2 presents a theoretical background of quantum information theory; the proposed algorithm is detailed in section 3. Finally, a set of experiments and comparisons are presented in section 4.

\section{Quantum information: Theoretical background}

In classical information theory, the basic information unit is the bit or binary digit which takes the value 0 or 1 , its correspondent in quantum information is the qubit. Contrary to the bit, the qubit can be presented as a superposition of 0 and 1 which represent in this case two orthogonal quantum eigenstates [24].

$$
|\psi\rangle=\alpha|0\rangle+\beta|1\rangle
$$

With: $|0\rangle=\left(\begin{array}{l}1 \\ 0\end{array}\right) ;|1\rangle=\left(\begin{array}{l}0 \\ 1\end{array}\right)$

This superposition can be written in matrix form as:

$$
|\psi\rangle=\left(\begin{array}{l}
\alpha \\
\beta
\end{array}\right)=\left(\begin{array}{l}
\alpha \\
0
\end{array}\right)+\left(\begin{array}{l}
0 \\
\beta
\end{array}\right)=\alpha\left(\begin{array}{l}
1 \\
0
\end{array}\right)+\beta\left(\begin{array}{l}
0 \\
1
\end{array}\right)
$$

With $|\psi\rangle$ represents the wave function, when $\alpha$ and $\beta$ are the probability amplitudes with two degrees of freedom each, satisfying:

$$
|\alpha|^{2}+|\beta|^{2}=1 \text {. }
$$


However, the normalization constraint (Equation 3) removes one degree of freedom. Thus, the geometric representation of the qubit was introduced by Bloch in 1964 [25] where the amplitudes are given by:

$$
\alpha=e^{i \gamma} \cos \frac{\theta}{2} ; \beta=e^{i(\phi+\gamma)} \sin \frac{\theta}{2}
$$

In the other side, the overall phase $e^{i \gamma}$ don't represent any physical identifiable consequences, we can therefore write the two amplitudes as:

$$
\alpha=\cos \frac{\theta}{2} ; \beta=e^{i \phi} \sin \frac{\theta}{2}
$$

With $\theta$ and $\phi$ represent the polar and azimuth angle of a quantum states respectively. These two angles can be used to map the qubit into a 3D spherical space so-called Bloch' sphere (Figure 1), equation (1) can be rewritten as:

$$
|\psi\rangle=\cos \frac{\theta}{2}|0\rangle+e^{i \phi} \sin \frac{\theta}{2}|1\rangle
$$

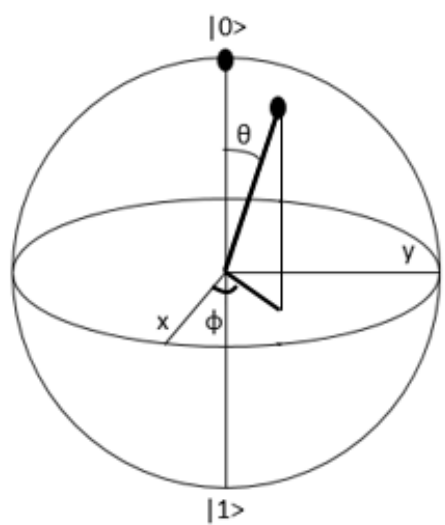

Figure 1: Bloch sphere.

Supposing we have a two qubits system, it has $2^{2}=4$ computational basis states denoted $\left|i_{b}\right\rangle=$ $\{|00\rangle,|01\rangle,|10\rangle,|11\rangle\}$. A pair of qubits can be at a superposition of these four states as:

$$
|\psi\rangle=\alpha_{00}|00\rangle+\alpha_{01}|01\rangle \alpha_{10}|01\rangle+\alpha_{11}|11\rangle
$$

Where $w_{4}=\left\{\alpha_{00}, \alpha_{01}, \alpha_{10}, \alpha_{11}\right\}$ are the amplitudes of the given system.

Similarly, in the case of a system of $n$ states, the equation 1 can be writing as:

$$
|\psi\rangle=\alpha_{1} \alpha_{2} \ldots \alpha_{n}|000 \ldots 0\rangle+\alpha_{1} \alpha_{2} \ldots \alpha_{n-1} \beta_{n}|00 \ldots 01\rangle+\ldots+\beta_{1} \beta_{2} \ldots \beta_{n}|111 \ldots 1\rangle
$$

And generalized as follow:

$$
|\psi\rangle=\sum_{i=1}^{2^{n}} w_{i}\left|i_{\text {binary }}\right\rangle
$$

Where $i_{\text {binary }}$ is an $n$-bits binary number and $w_{i}$ is the probability of the $i^{\text {th }}$ state satisfying:

$$
\sum_{i=1}^{2^{n}}\left|w_{i}\right|^{2}=1
$$

Based on the above descriptions quantum image processing can be modelled using the basic representation of qubit.

Let $I(x, y)$ be the intensity value of a normalized grey scale image pixel at the $(\mathrm{x}, \mathrm{y})$ position. the qubit pixel can be obtained according to the equation 1 and the eigenstates constraint, under the viewpoint of the probability statistics by: 


$$
|I(x, y)\rangle=\sqrt{1-I(x, y)}|0\rangle+\sqrt{I(x, y)}|1\rangle
$$

Where $\sqrt{1-I(x, y)}$ and $\sqrt{I(x, y)}$ are the probabilities of having the current pixel at the state 0 and 1 respectively, and satisfying $|\sqrt{1-I(x, y)}|^{2}+|\sqrt{I(x, y)}|^{2}=1$.

\section{Proposed Method}

\subsection{Spherical neighborhood on Bloch's sphere}

The principal limitation of using classical edge detectors directly on omnidirectional image is that whatever the kernels position in the image, its effect on the pixel's neighborhood remains the same leading to false edge detection (since the neighborhood definition is different in the case of omnidirectional image).

In order to solve this issue, and as we are working in the QIP, we propose to work with an equivalent image based on Bloch' sphere where each pixel is defined by its intensity $I$ and its position on the Bloch's Sphere according to the polar and azimuth angles $\theta$ and $\phi$ as $I(\theta, \phi)$ instead of planar 2D position $\mathrm{x}$ and $\mathrm{y}$ as $I(x, y)$.

Let $\psi_{\text {Sphere }}^{i}$ be the $i^{\text {th }}$ pixel in the spherical Moore neighborhood and $I_{i}$ its intensity. Based on the equations 5, 6 and 11 and taking into account the Bloch's sphere, we propose an adapted pixel quantum state, which bring together both intensity-based state and the one based on the spherical position on Bloch's sphere, as follow:

The wave function is presented in the Bloch's sphere by: $|\psi\rangle=\alpha|0\rangle+\beta|1\rangle$ where $\alpha=\cos \frac{\theta}{2} ; \beta=e^{i \phi} \sin \frac{\theta}{2}(\theta$ and $\phi$ are the polar and azimuth angle of the quantum pixel respectively). In addition, the qubit pixel is given by: $|I(x, y)\rangle=\sqrt{1-I(x, y)}|0\rangle+\sqrt{I(x, y)}|1\rangle$, where $I(x, y)$ is the intensity value of the pixel at $(\mathrm{x}, \mathrm{y})$ position. The main idea is to have the qubit pixel with polar coordinates, we shall introduce the adapted pixel quantum state as:

$$
\left|\psi_{\text {Sphere }}^{i}\right\rangle=A_{i} \cdot|0\rangle+B_{i} \cdot|1\rangle \quad i=1,2, \ldots, 6
$$

Where:

$$
\begin{gathered}
A_{i}=\frac{\sqrt{1-I_{i}(\theta, \phi)}}{\alpha_{i}^{2}} ; B_{i}=\frac{\sqrt{I_{i}(\theta, \phi)-\beta_{i}^{2}}}{1-\beta^{2}} \\
\alpha_{i}=\cos \frac{\theta_{i}}{2} ; \beta_{i}=e^{i \phi} \sin \frac{\theta_{i}}{2} ; \theta \epsilon\left[0: \frac{\pi}{2}[; \phi=[0: 2 \pi[\right.
\end{gathered}
$$

The amplitudes $A_{i}$ and $B_{i}$ satisfies the condition presented in the equation 3:

$$
\left|\frac{\sqrt{1-I_{i}(\theta, \phi)}}{\alpha_{i}^{2}}\right|^{2}+\left|\frac{\sqrt{I_{i}(\theta, \phi)-\beta_{i}^{2}}}{1-\beta^{2}}\right|^{2}=1
$$

\subsection{Object edges enhancement by quantum superposition}

The next step in our process of edge detection is to get a more accentuated object edges, in order to avoid false detections comes from a textured background, we consider the Moore neighborhood of the active pixel as illustrated in Figure 2: 


\begin{tabular}{|l|l|l|}
\multicolumn{2}{c}{} & \multicolumn{2}{c}{$\square$ Active pixel } \\
\hline$\psi_{\text {Sphere }}^{5}$ & $\psi_{\text {Sphere }}^{6}$ & $\psi_{\text {Sphere }}^{7}$ \\
\hline$\psi_{\text {Sphere }}^{4}$ & $\psi_{\text {Sphere }}^{1}$ & $\psi_{\text {Sphere }}^{8}$ \\
\hline$\psi_{\text {Sphere }}^{3}$ & $\psi_{\text {Sphere }}^{2}$ & $\psi_{\text {Sphere }}^{9}$ \\
\hline
\end{tabular}

Figure 2: the Moore neighborhood of the active pixel.

For a better result, we enhance the edge points in four different directions in each Moore neighborhood:

- $\left\langle\psi_{\text {Sphere }}^{4}\left|\psi_{\text {Sphere }}^{1}\right| \psi_{\text {Sphere }}^{8}\right\rangle$ as the $0^{\circ}$ direction.

- $\left\langle\psi_{\text {Sphere }}^{3}\left|\psi_{\text {Sphere }}^{1}\right| \psi_{\text {Sphere }}^{7}\right\rangle$ as the $45^{\circ}$ direction.

- $\left\langle\psi_{\text {Sphere }}^{2}\left|\psi_{\text {Sphere }}^{1}\right| \psi_{\text {Sphere }}^{6}\right\rangle$ as the $90^{\circ}$ direction.

- $\left\langle\psi_{\text {Sphere }}^{9}\left|\psi_{\text {Sphere }}^{1}\right| \psi_{\text {Sphere }}^{5}\right\rangle$ as the $135^{\circ}$ direction.

Then, we apply equations 9 and 12 for each subsystem of three pixels, an example for the $90^{\circ}$ direction is given in the Table 1, where the state ' 0 ' and ' 1 ' represent a background and an object pixel respectively.

Table 1: Different states of the $90^{\circ}$ direction.

\begin{tabular}{cccc}
\hline & $\boldsymbol{i}_{\boldsymbol{b}}$ & & $\boldsymbol{w}_{\boldsymbol{i}}$ \\
\hline 0 & 0 & 0 & $A_{2} \cdot A_{1} \cdot A_{6}$ \\
0 & 0 & 1 & $A_{2} \cdot A_{1} \cdot B_{6}$ \\
0 & 1 & 0 & $A_{2} \cdot B_{1} \cdot A_{6}$ \\
0 & 1 & 1 & $A_{2} \cdot B_{1} \cdot B_{6}$ \\
1 & 0 & 0 & $B_{2} \cdot A_{1} \cdot A_{6}$ \\
1 & 0 & 1 & $B_{2} \cdot A_{1} \cdot B_{6}$ \\
1 & 1 & 0 & $B_{2} \cdot B_{1} \cdot A_{6}$ \\
1 & 1 & 1 & $B_{2} \cdot B_{1} \cdot B_{6}$ \\
\hline
\end{tabular}

By definition, an edge point (edge pixel) is defined as an object pixel that have at least one background pixel in its neighborhood [26], so this two cases can be ignored:

Case 1: The processed pixel is a background pixel.

Case 2: The processed pixel is surrounded only by object pixels.

By ignoring these two cases, and for each subsystem in a given direction, we have:

$$
\begin{gathered}
\left\langle\psi_{\text {Sphere }}^{2}\left|\psi_{\text {Sphere }}^{1}\right| \psi_{\text {Sphere }}^{6}\right\rangle=A_{2} \cdot B_{1} \cdot A_{6}|010\rangle+A_{2} \cdot B_{1} \cdot B_{6}|011\rangle+B_{2} \cdot B_{1} \cdot A_{6}|110\rangle \\
\operatorname{Prob}_{90^{\circ}}=\sum w_{i} \mid i \in\{2,3,6\}=B_{1} \cdot\left(B_{2} A_{6}+A_{2} \cdot\left(A_{6}+B_{6}\right)\right)
\end{gathered}
$$

Similarly, we have:

$$
\begin{aligned}
& \operatorname{Prob}_{0^{\circ}}=B_{1} \cdot\left(B_{4} A_{8}+A_{4} \cdot\left(A_{8}+B_{8}\right)\right) \\
& \operatorname{Prob}_{45^{\circ}}=B_{1} \cdot\left(B_{3} A_{7}+A_{3} \cdot\left(A_{7}+B_{7}\right)\right) \\
& \operatorname{Prob}_{135^{\circ}}=B_{1} \cdot\left(B_{9} A_{5}+A_{9} \cdot\left(A_{5}+B_{5}\right)\right)
\end{aligned}
$$

After that, the processed pixel is replaced by the maximum value between the 4 directions as:

$$
\operatorname{Pixel}(\theta, \phi)=\max \left\{\operatorname{Prob}_{0^{\circ}}, \operatorname{Prob}_{45^{\circ}}, \operatorname{Prob}_{90^{\circ}}, \operatorname{Prob}_{135^{\circ}}\right\}
$$


Each new enhanced pixel $\operatorname{Pixel}(\theta, \phi)$ is processed according the described steps in order to generate a new enhanced image.

\subsection{Edge Localization by quantum entropy}

The concept of Quantum entropy was introduced the first time by John von Neumann in 1932 [27] which represent a quantum generalization of the classical Shannon entropy and gives meaning to the quantum information theory in a qubit. This concept can be presented as the measure of the amount of information, uncertainty or disorder in a quantity of information, it's given by:

$$
S_{\text {quantum }}=-\operatorname{Trace}(\rho \log \rho)
$$

Where $\rho$ is the density matrix of the system.

Many edge detection algorithms have been developed based on entropy theory [28]-[31]. We applied the local quantum entropy in each Moore neighborhood of the processed image, a higher entropy, correspond to an edge pixel. For each central pixel of the Moore neighborhood, its defined as an edge pixel if the local entropy is greater than a threshold $H_{L}$ funded practically.

$$
\operatorname{Pixel}(\theta, \phi)= \begin{cases}1 & \text { if } H_{L}<S_{\text {quantum }} \\ 0 & \text { if } H_{L}>S_{\text {quantum }}\end{cases}
$$

The proposed algorithm is summarized in the following steps:

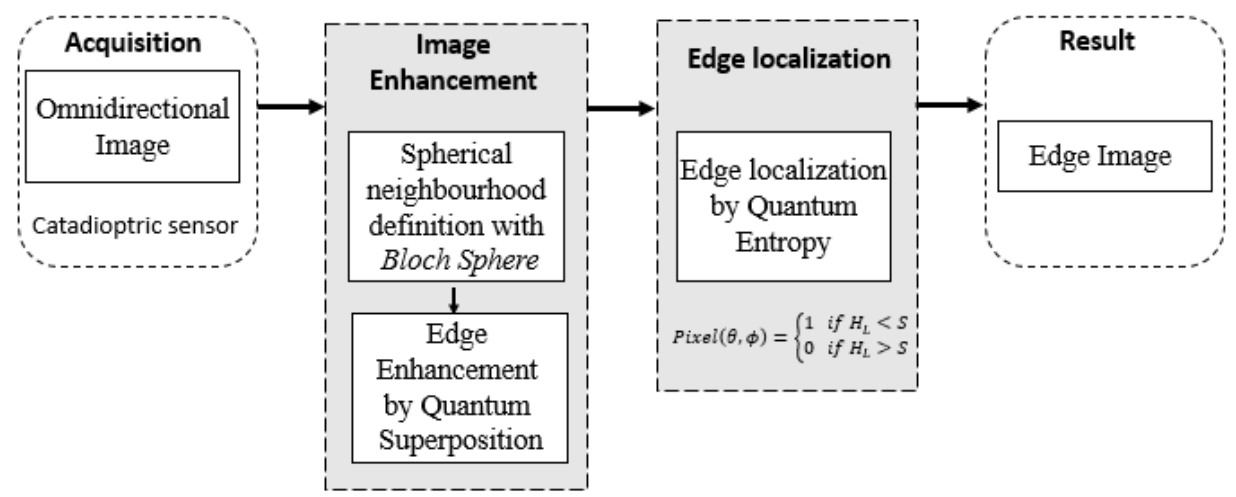

Figure 3: Summary of the proposed algorithm.

\section{Experiment results and discussion}

In this section, a set of experimentations is done, on synthetic and real images, in order to show the algorithm performance in term of edge continuity and behavior against noise, compared to other algorithms presented for the same purpose.

\subsection{Evaluation method}

In order to evaluate the proposed method in term of edge detection quality (edge continuity and behavior to noise), a measurable criterion is needed. In [23], Fram \& Deutsh presented two criterions $P_{1}$ and $P_{2}$ which measures the detector behaviour against noise and edge continuity respectively, ranges between 0 (poor behavior) and 1 (good behavior), and applied to blocks of $36 \times 36$ containing 3 regions, as shown at the Figure 4. 


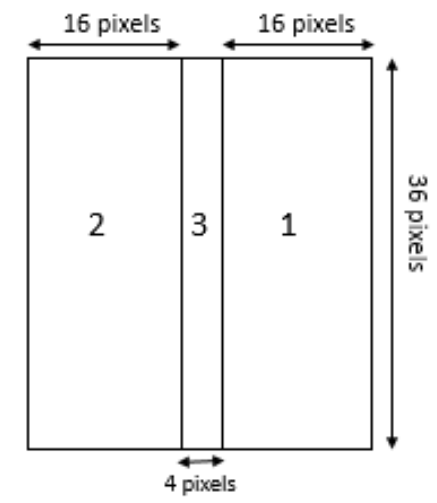

Figure 4: The 3 regions of the Fram \& Deutsh block test.

The Figure 5 represents an example of the Fram \& Deutsh parameters extraction from a synthetic omnidirectional image with two regions. Let $C$ be the image region taking into consideration and containing two parts: $C_{e}$ the outside edge region and $C_{i}$ the inside edge region (Figure 5). Accordingly, $w_{1}^{e}$ is the number of columns in the edge region $C_{i} . n^{e}$ and $n^{0}$ represent the number of detected edge pixels inside and outside $C_{i}$ respectively. $w_{1}$ and $w_{2}$ are the number of columns and rows of the output binary image respectively, $n^{e}$ and $n^{0}$ represent the number of detected edge pixels inside and outside of the edge zone respectively, $w_{1}^{\text {stan }}$ is by default set to 30 and to finish $n^{r}$ is the number of rows in the edge region that contain at least one detected edge point. The $P_{1}$ and $P_{2}$ are given by:

$$
P_{1}=\frac{n_{\text {sig }}^{e}}{n_{\text {sig }}^{e}+\left(n_{\text {noise }}^{e}+n^{0}\right)\left(w_{1}^{\text {stan }} / w_{1}\right)}
$$

where

$$
n_{\text {sig }}^{e}=\frac{n^{e}-n_{\text {noise }}^{e}}{1-n_{\text {noise }}^{e} / w_{1}^{e} w_{2}}
$$

and

$$
\begin{gathered}
n_{\text {noise }}^{e}=n^{0} \frac{w_{1}^{e}}{w_{1}-w_{1}^{e}} \\
P_{2}=\frac{n^{r} / w_{2}-\left\{1-\left[1-n_{\text {noise }}^{e} / w_{1}^{e} w_{2}\right]_{1}^{w_{1}^{e}}\right.}{\left[1-\left(n_{\text {noise }}^{e} / w_{1}^{e} w_{2}\right)\right]^{w_{1}^{e}}}
\end{gathered}
$$

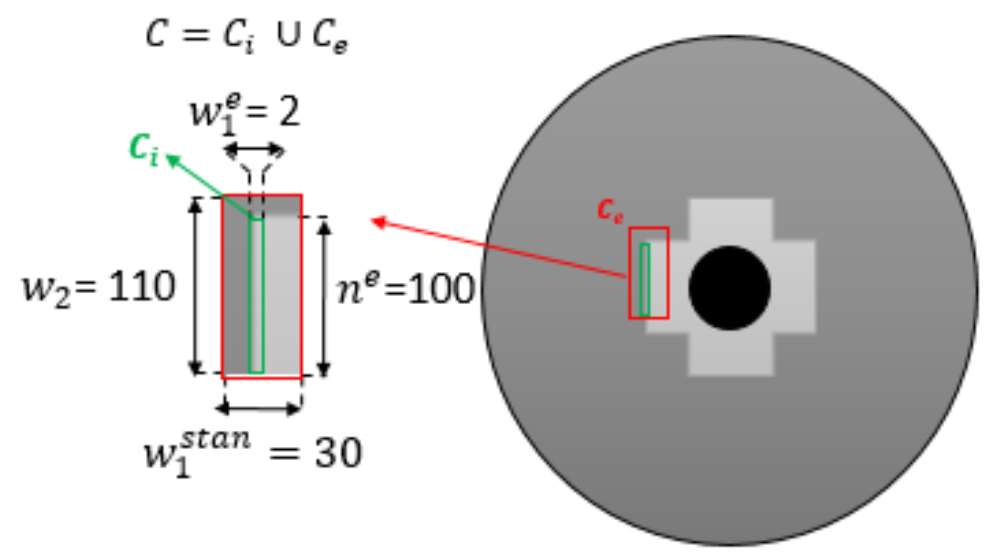

Figure 5: An example of Fram \& Deutsh parameters for a synthetic omnidirectional image. 


\subsection{Materials}

Our goal is the segmentation of omnidirectional images contours. Therefore, we used two types of images: synthetic images for the method evaluation and comparisons, and real images taking from an omnidirectional camera inside a greenhouse, where we used a calibrated omnidirectional camera composed of a spherical mirror and perspective camera, in a face-to-face configuration (Figure 6). The Table $2 \&$ Table 3 contains the details about the omnidirectional camera and the used images.

Table 2: Omnidirectional camera details

\begin{tabular}{cccc}
\hline & Mirror & \multicolumn{3}{c}{ Omnidirectional camera } \\
\hline Type & Spherical & Sensor type & CCD $-23.0 \times 15.5 \mathrm{~mm}$ \\
Radius & $3 \mathrm{~cm}$ & Focal length $(\mathbf{m m})$ & 28.4 \\
Focal length & $\sim 1.5 \mathrm{~cm}$ & Resolution $($ Pixels) & $4256 \times 2848$ \\
Material & Stainless steel & Pixel size $(\mathbf{m m})$ & 0.0054 \\
\end{tabular}

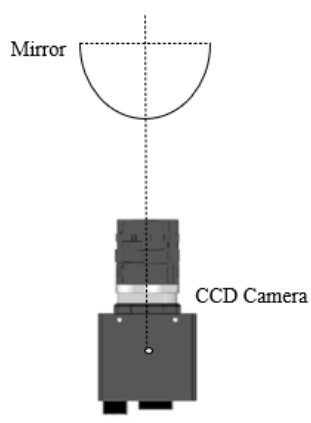

Figure 6: Face to face configuration of the used omnidirectional camera

Table 3: Details of the used images

\section{Synthetic images}

Used for

Number

Format

Original type

Resolution

(Pixels) Group)

Grayscale

$800 \times 400$

\section{Real images}

Agricultural application

5

TIFF (Tagged Image File Format)

RGB

$4256 \times 2848$ 


\subsection{Synthetic images}

In order to evaluate the performance of the proposed method, a comparison against three methods proposed in the literature is performed: i) The method proposed by Guelzim \& al, which propose and edge detection algorithm for omnidirectional images based on four steps: a) Image quantification b) calculation of the entropy $\mathrm{H}$ matrix c) local maxima verification and d) thresholding [10]. ii) The method proposed by Jacquey \& al which estimate image gradient based on non-additive approach [32] and iii) The method proposed by EL kadmiri \& al that used a spherical virtual electrostatic charges distribution model to detect edges in spherical images [33]. We applied those methods in three representative synthetics omnidirectional images devoted to edge detection applications and contain different type of edges. Also, Gaussian and Salt \& Pepper noises are added to those images in order to evaluate the behavior of the tested methods against noise.

As described before, the second step of the proposed method is the edge enhancement, which is performed using quantum superposition laws on the Bloch sphere, the Figure 7 Shows the edge enhancement for an omnidirectional synthetic image, where some regions of the synthetic image were zoomed to show the edge enhancement in different image parts. Figure 8 and Figure 9 shows the application of the considered edge detectors for the synthetic omnidirectional images.

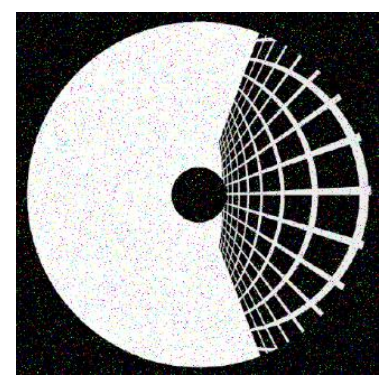

(a)

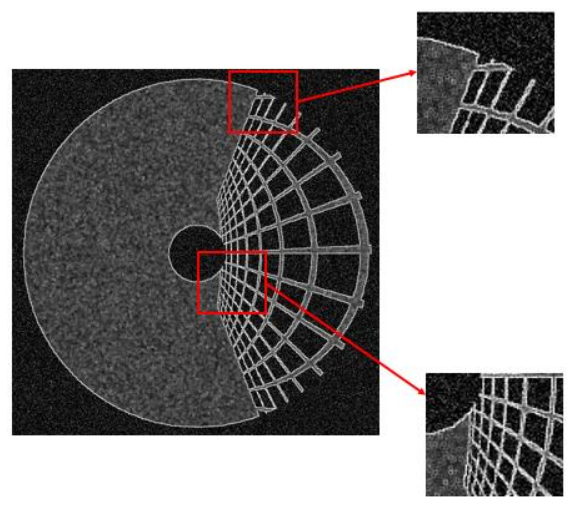

(b)

Figure 7: (a) Original noised synthetic image. (b) Edge enhancement for the synthetic image.
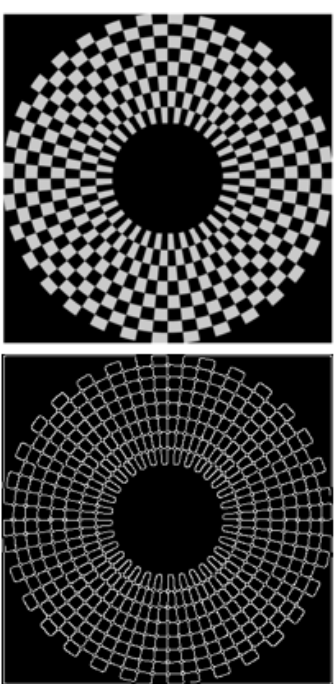

(a)
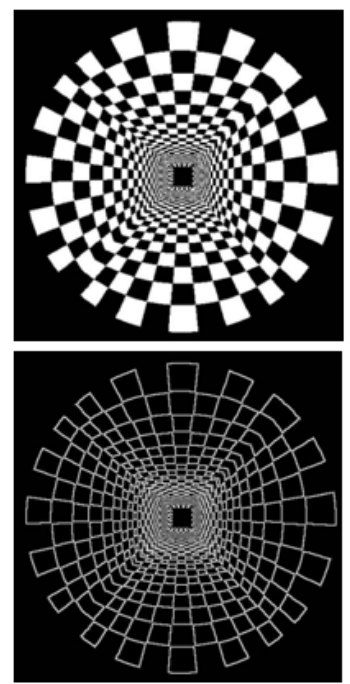

(b)
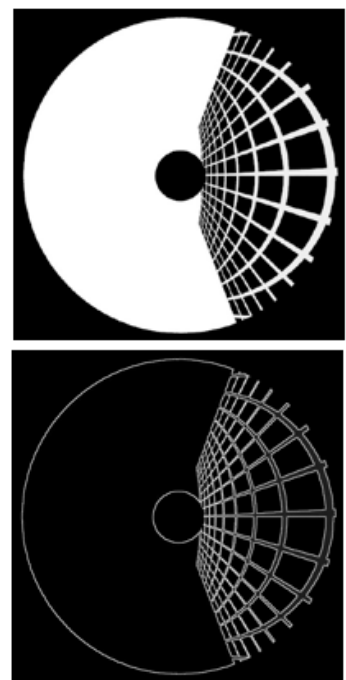

(c)

Figure 8: Edge detection using the proposed method for three synthetic omnidirectional images. 
The first synthetic omnidirectional image (Figure 8.a) contains a black and white checkboard in a white background, the checkboard presents a radial distortion which reduce the resolution near the center which generates a line deformation. The same, the second (Figure 8.b) and third image (Figure 8.c) contain a $360^{\circ}$ checkboard which present a good example of the radial distortion generated by the spherical mirror, these two images simulate a catadioptric camera placed inside a cylinder and a parallelepiped respectively.

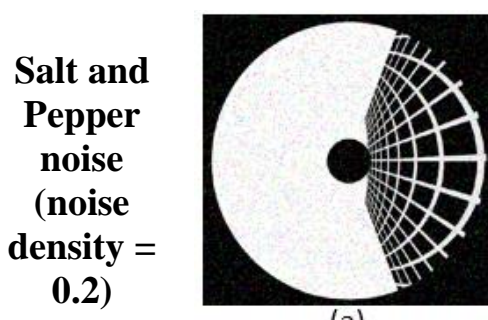

(a)

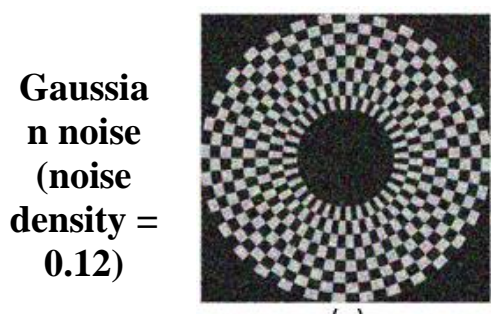

(a)

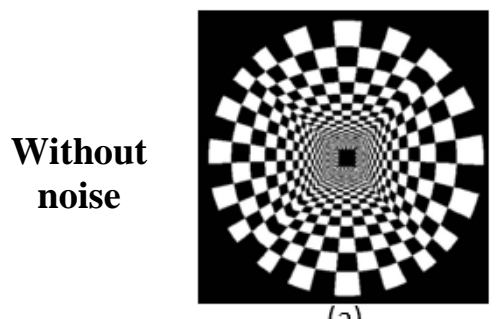

(a)

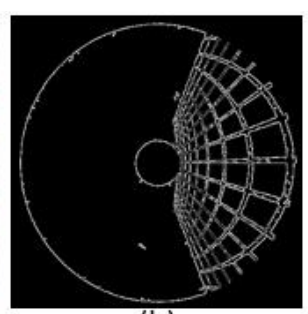

(b)

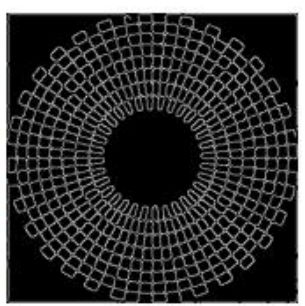

(b)

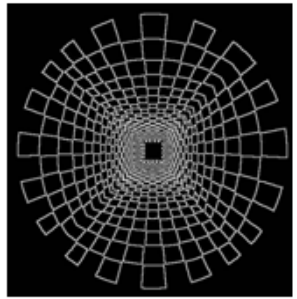

(b)

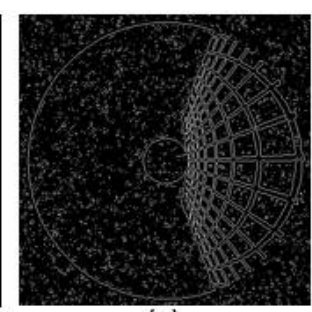

(c)

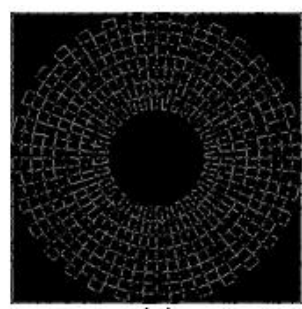

(c)

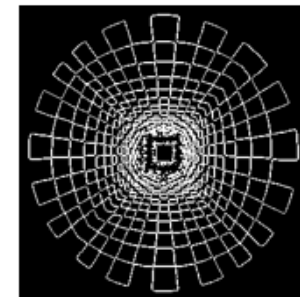

(c)

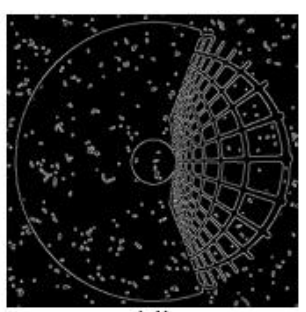

(d)

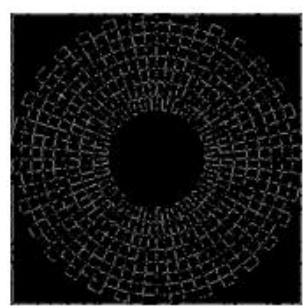

(d)

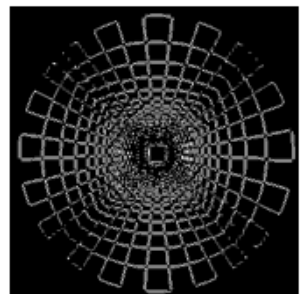

(d)

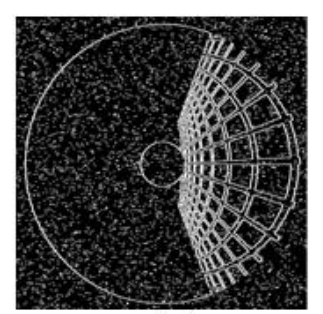

(e)

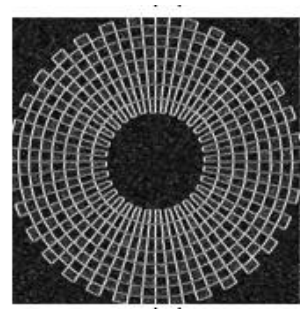

(e)

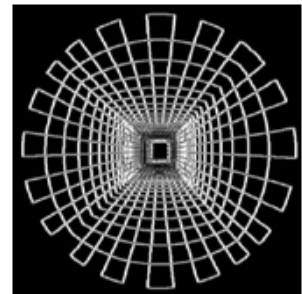

(e)

Figure 9: Application of different edge detection method to the three synthetic images. (a) Original synthetic image. (b) Proposed method. (c) Guelzim and al [10] method. (d) Jacquey \& al [32] method. (e) EL kadmiri $\&$ al [33]

As a quantifiable measurement of the methods performance, Table 4 and Table 5 presents $P_{1}$ and $P_{2}$ values for the tested synthetic image in different cases: without noise, with Gaussian noise and with Salt and Pepper.

Table 4: $P_{1}$ values of the different tested algorithms.

\begin{tabular}{cccc}
\cline { 2 - 4 } & Without noise & Gaussian noise & Salt \& Pepper noise \\
\hline Proposed algorithm & $\mathbf{0 . 9 1 2 1}$ & $\mathbf{0 . 8 9 1 9}$ & $\mathbf{0 . 9 0 0 9}$ \\
Guelzim \& al & 0.8380 & 0.8240 & 0.8314 \\
Jacquey \& al & 0.8802 & 0.8711 & 0.8699 \\
EL kadmiri \& al & 0.8902 & 0.8800 & 0.8107 \\
\hline
\end{tabular}


Table 5: $P_{2}$ values of the different tested algorithms.

\begin{tabular}{cccc}
\cline { 2 - 4 } & Without noise & Gaussian noise & Salt \& Pepper noise \\
\hline Proposed algorithm & 0.9891 & $\mathbf{0 . 9 6 3 8}$ & $\mathbf{0 . 9 7 7 0}$ \\
Guelzim \& al & 0.9329 & 0.9100 & 0.8129 \\
Jacquey \& al & 0.9891 & 0.9190 & 0.8591 \\
EL kadmiri \& al & $\mathbf{0 . 9 8 9 4}$ & 0.9209 & 0.9609 \\
\hline
\end{tabular}

Since the criterion $P_{1}$ measure the robustness of the edge detection method against noise, the Table 4 show that the proposed method is resistant to different type of noise compared to the other tested methods. On the other hand, the Table 5 show the values of the $P_{2}$ which characterise the performance of the method in term of edge continuity. These two parameters show that the proposed method present a better performance in both the edge continuity and sensibility to noise.

\subsection{Real omnidirectional images devoted to agricultural robotics.}

In order to validate the results from the previous section, we applied the proposed method to omnidirectional images captured in Strawberry greenhouse. For this, we used the omnidirectional camera resented in the materials section.

The Figure 10 show two omnidirectional images in a strawberry greenhouse for robotics experimentations, taking from an embedded omnidirectional camera in the agricultural robot we presented in [34]. We used this type of images for our robot navigation between the greenhouse' rows. For each acquired image, the edge map is generated using the proposed method (Figure 11), then the deviation of the robot (Green line) is calculated using the center axis between the rows as a reference (Red line)

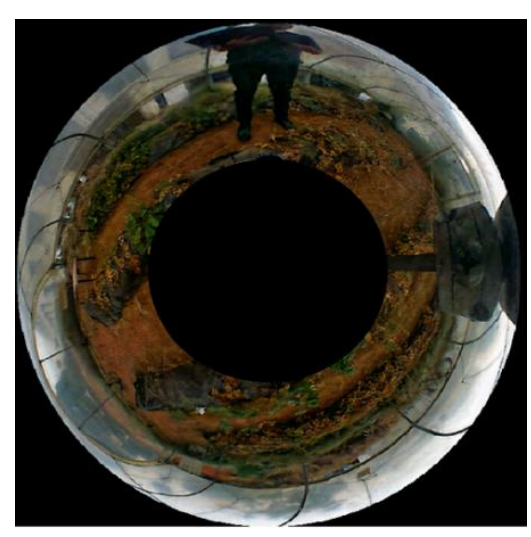

(a)

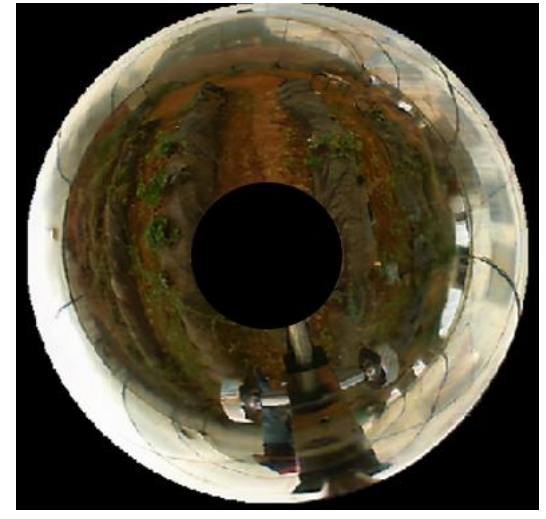

(b)

Figure 10: Omnidirectional images of a strawberry greenhouse. 

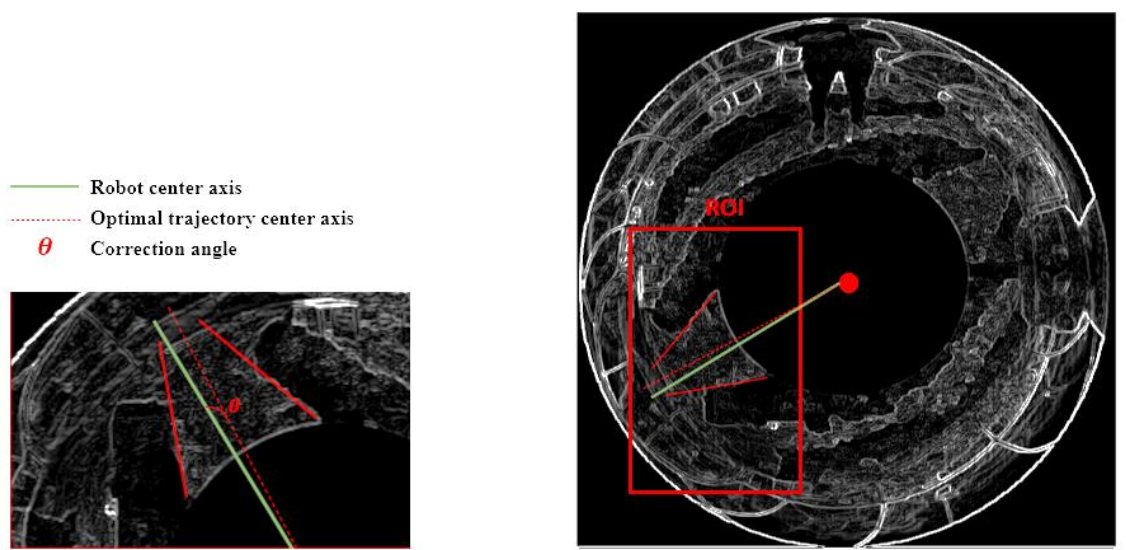

Figure 11: Path estimation using edge detection in omnidirectional image.

\section{Conclusion}

In this paper, we present an algorithm for edge detection in omnidirectional images, based on quantum entropy and superposition laws on Bloch's sphere. The proposed method has the advantage of being developed directly on the spherical space, which make it adapted to the spherical images by taking into account its geometry, and being non-gradient based, which positively affect its robustness against different type of noise. These advantages have been shown through the Fram \& Deutsh criterions, where the proposed method gives a better performance compared to the other tested methods in both edge continuity and sensibility to noise. In addition, we observed that the computational time can be improved and more optimal outcomes can be attained with the implementation with a deep learning approach. As a future work, we encourage to use the proposed algorithm for point of interest detection in omnidirectional images. The proposed edge detection algorithm can be used in various applications and devises such as: robots, quality control systems, UAV applications and authentication systems.

\section{ACKNOWLEDGEMENT}

The authors of this paper are thankful to the Ministry of Higher Education and Scientific Research of Morocco (MESRSFC), and the National Centre of Scientific and Technical Research of Morocco (CNRST) for financing this project.

\section{REFERENCES}

[1] M. Boui, "Détection et suivi de personnes par vision omnidirectionnelle : approche 2D et 3D," PhD Thesis, Paris-Saclay University (ComUE), 2018.

[2] L. F. Posada, A. Velasquez-Lopez, F. Hoffmann, and T. Bertram, "Semantic Mapping with Omnidirectional Vision," in 2018 IEEE International Conference on Robotics and Automation (ICRA), May 2018, pp. 1901-1907, doi: 10.1109/ICRA.2018.8461165.

[3] M. Vlaminck, H. Luong, W. Goeman, and W. Philips, "3D Scene Reconstruction Using Omnidirectional Vision and LiDAR: A Hybrid Approach," Sensors, vol. 16, no. 11, Art. no. 11, Nov. 2016, doi: 10.3390/s16111923.

[4] J. Bermudez-Cameo, "New contributions on line-projections in omnidirectional vision," ELCVIA: electronic letters on computer vision and image analysis, vol. 15, no. 2, pp. 24-26, 2016, doi: 10.5565/rev/elcvia.948.

[5] S. Peleg and M. Ben-Ezra, "Stereo panorama with a single camera," in Proceedings. 1999 IEEE Computer Society Conference on Computer Vision and Pattern Recognition (Cat. No PR00149), Fort Collins, CO, USA, 1999, pp. 395-401, doi: 10.1109/CVPR.1999.786969. 
[6] I. Powell, "Panoramic fish-eye imaging system," US Patent, $\mathrm{N}^{\circ}: 5,631,778$.

[7] S. K. Nayar, "Sphereo: Determining Depth Using Two Specular Spheres And A Single Camera," in Optics, Illumination, and Image Sensing for Machine Vision III, Mar. 1989, vol. 1005, pp. 245-254, doi: 10.1117/12.949051.

[8] S. Barone, P. Neri, A. Paoli, and A. V. Razionale, "Catadioptric stereo-vision system using a spherical mirror," Procedia Structural Integrity, vol. 8, pp. 83-91, Jan. 2018, doi: 10.1016/j.prostr.2017.12.010.

[9] Q. Shi, C. Li, C. Wang, H. Luo, Q. Huang, and T. Fukuda, "Design and implementation of an omnidirectional vision system for robot perception," Mechatronics, vol. 41, pp. 58-66, Feb. 2017, doi: 10.1016/j.mechatronics.2016.11.005.

[10] I. Guelzim, "Contributions aux traitements d'images perspectives et omnidirectionnelles par des outils statistiques.," PhD Thesis, Mohammed V University, Rabat, Morocco, 2012.

[11] Eder, M., \& Frahm, J. M. (2019). Convolutions on spherical images. In Proceedings of the IEEE/CVF Conference on Computer Vision and Pattern Recognition Workshops (pp. 1-5).

[12] J. Li, Z. Wen, S. Li, Y. Zhao, B. Guo, and J. Wen, "Novel tile segmentation scheme for omnidirectional video," in 2016 IEEE International Conference on Image Processing (ICIP), Sep. 2016, pp. 370-374, doi: 10.1109/ICIP.2016.7532381.

[13] Z. Kerkaou, N. Alioua, M. E. Ansari, and L. Masmoudi, "Edge points-based stereo matching approach for omnidirectional images," JEI, vol. 27, no. 5, p. 053015, Sep. 2018, doi: 10.1117/1.JEI.27.5.053015.

[14] F. Ababsa, H. Hadj-Abdelkader, and M. Boui, "3D Human Tracking with Catadioptric Omnidirectional Camera," in Proceedings of the 2019 on International Conference on Multimedia Retrieval, Ottawa ON Canada, Jun. 2019, pp. 73-77, doi: $10.1145 / 3323873.3325027$.

[15] K. Bhongale and S. Gore, "Design of robot navigation monitoring system using image feature analysis and omnidirectional camera images," In 2017 2nd International Conference for Convergence in Technology (I2CT) IEEE, pp. 405-409, doi: 10.1109/I2CT.2017.8226161

[16] K. Qiu, T. Liu, and S. Shen, "Model-Based Global Localization for Aerial Robots Using Edge Alignment," IEEE Robot. Autom. Lett., vol. 2, no. 3, pp. 1256-1263, Jul. 2017, doi: 10.1109/LRA.2017.2660063.

[17] S. Li, "Spherical gradient operator: SPHERICAL GRADIENT OPERATOR," IEEJ Trans Elec Electron Eng, vol. 8, no. S1, pp. S61-S65, 2013, doi: 10.1002/tee.21919.

[18] C. Demonceaux, P. Vasseur, and Y. Fougerolle, "Central catadioptric image processing with geodesic metric," Image and Vision Computing, vol. 29, no. 12, pp. 840-849, Nov. 2011, doi: 10.1016/j.imavis.2011.09.007.

[19] C. Demonceaux and P. Vasseur, "Markov random fields for catadioptric image processing," Pattern Recognition Letters, vol. 27, no. 16, pp. 1957-1967, Dec. 2006, doi: 10.1016/j.patrec.2006.05.007.

[20] A. Makadia, C. Geyer, and K. Daniilidis, "Correspondence-free Structure from Motion," Int J Comput Vis, vol. 75, no. 3, pp. 311-327, Dec. 2007, doi: 10.1007/s11263-007-0035-2.

[21] K. Hara, K. Inoue, and K. Urahama, "Gradient operators for feature extraction from omnidirectional panoramic images," Pattern Recognition Letters, vol. 54, pp. 89-96, Mar. 2015, doi: 10.1016/j.patrec.2014.12.010.

[22] J. Watrous, Theory of quantum information. Cambridge University Press, 2018.

[23] J. R. Fram and E. S. Deutsch, "On the Quantitative Evaluation of Edge Detection Schemes and their Comparison with Human Performance," IEEE Transactions on Computers, vol. C-24, no. 6, pp. 616-628, Jun. 1975, doi: 10.1109/T-C.1975.224274.

[24] K. Fujii, Quantum Computation with Topological Codes: From Qubit to Topological FaultTolerance, vol. 8. Singapore: Springer Singapore, 2015.

[25] Bloch, F. (1946). Nuclear induction. Physical review, 70(7-8), 460, 1946. 
[26] A. Ezzaki, M. Lhoussaine, M. E. Ansari, F.-A. Moreno, R. Zenouhi, and J. G. Jimenez, "Edge detection algorithm based on quantum superposition principle and photons arrival probability," International Journal of Electrical \& Computer Engineering, vol. 10, no. 2, p. 12, 2020. doi: 10.11591/ijece.v10i2.pp1655-1666

[27] J. Von Neumann, R. T. Beyer, and N. A. Wheeler, Mathematical foundations of quantum mechanics, New edition. Princeton: Princeton University Press, 2018.

[28] V. Rajinikanth and S. C. Satapathy, "Segmentation of Ischemic Stroke Lesion in Brain MRI Based on Social Group Optimization and Fuzzy-Tsallis Entropy," Arabian Journal for Science and Engineering, Jan. 2018, doi: 10.1007/s13369-017-3053-6.

[29] M. A. El-Sayed and T. A.-E. Hafeez, "New edge detection technique based on the shannon entropy in gray level images," arXiv preprint arXiv:1211.2502, 2012.

[30] B. Singh and A. P. Singh, "Edge Detection in Gray Level Images based on the Shannon Entropy 1," Journal of Computer Science, pp. 186-191, 2008.

[31] M. A. El-Sayed and H. A. M. Sennari, "Multi-Threshold Algorithm Based on Havrda and Charvat Entropy for Edge Detection in Satellite Grayscale Images," Journal of Software Engineering and Applications, vol. 07, no. 01, pp. 42-52, 2014, doi: 10.4236/jsea.2014.71005.

[32] F. Jacquey, F. Comby, and O. Strauss, "Non-additive Approach for Omnidirectional Image Gradient Estimation," in 2007 IEEE 11th International Conference on Computer Vision, Rio de Janeiro, Brazil, 2007, pp. 1-6, doi: 10.1109/ICCV.2007.4409193.

[33] O. E. Kadmiri, Z. E. Kadmiri, and L. Masmoudi, "A spherical electrostatic model edge detector for omnidirectional images," Journal of Theoretical and Applied Information Technology, vol. 51, p. 9, 2013.

[34] A. Abanay, Lh. Masmoudi, A. Elharif, M. Gharbi, and B. Bououlid, "Design and development of a mobile platform for an agricultural robot prototype," in Proceedings of the 2nd International Conference on Computing and Wireless Communication Systems - ICCWCS'17, Larache, Morocco, 2017, pp. 1-5, doi: 10.1145/3167486.3167527. 